\title{
ON THE NOTION OF REGULARITY OF METHODS OF SUMMATION OF INFINITE SERIES
}

\author{
BY J. D. TAMARKIN*
}

Let $\mathfrak{A}=\left(a_{m n}\right)$ be the matrix of a method of summation which consists in replacing a given sequence $x=\left(x_{1}, x_{2}, \cdots\right)$ by its transform

(1) $y=\left(y_{1}, y_{2}, \cdots\right), \quad y_{m}=\sum_{n=1}^{\infty} a_{m n} x_{n}, \quad(m=1,2, \cdots)$,

and defining the generalized limit of $x_{n}$ as $\lim _{m \rightarrow \infty} y_{m}$, provided this limit exists.

The method $\mathfrak{A}$ is called regular if every convergent sequence $x=\left\{x_{n}\right\}$ is transformed into a convergent sequence $y=\left\{y_{m}\right\}$ with the same limit. Necessary and sufficient conditions for regularity of $\mathfrak{A}$ are too well known to be restated here. An essential point in the whole theory is the assumption that the sequence $y \equiv y(x)$ is determined by formula (1) for each convergent sequence $x$. A (quite trivial) gain in generality may be achieved by demanding that not all the terms $y_{m}$ of the sequence $\left\{y_{m}\right\}$ have to be considered but only those for which $m \geqq m_{0}$, where $m_{0}$ is a fixed integer. Even this requirement is not at all necessary, however, for the possibility of evaluating $\lim _{m \rightarrow \infty} y_{m}$, for which we have to know only almost all terms of the sequence $\left\{y_{m}\right\}$, that is all $y_{m}, m \geqq m^{\prime}$, where $m^{\prime}$ need not be fixed, but on the contrary, may depend on the sequence $x$.

Thus we are naturally led to the following apparently less restrictive definition of regularity of the method of summation $\mathfrak{A}$.

The method of summation $\mathfrak{A}$ is regular if (i) to every convergent sequence $x=\left\{x_{n}\right\}$ there corresponds an integer $m^{\prime}(x)$ such that $y_{m}$ as given by (1) exists for $m \geqq m^{\prime}(x)$, and (ii) for a fixed $x$,

$$
\lim _{m \rightarrow \infty} y_{m}=\lim _{n \rightarrow \infty} x_{n} \text {. }
$$

It turns out, however, that the modified definition of regularity

* The result of this note answers a question raised by Dr. H. Lewy. 
actually is not more general than the classical one. Indeed we intend to show that if $\mathfrak{U}$ satisfies condition (i) above, then there exists a fixed number $m_{0}$ which does not depend on $x$, such that all the series

$$
y_{m}=\sum_{n=1}^{\infty} a_{m n} x_{n}, \quad\left(m \geqq m_{0}\right)
$$

converge.

Assume that the number in question does not exist. Then we can find a sequence of integers $\left\{m_{1}, m_{2}, \cdots\right\}, m_{p} \rightarrow \infty$ and a set of sequences $x^{(p)}=\left\{x_{n}{ }^{(p)}\right\}$, such that none of the series

$$
\sum_{n=1}^{\infty} a_{m n} x_{n}^{(p)}, \quad\left(m=m_{p} ; p=1,2, \cdots\right),
$$

converges. Now introduce the space (c) of all convergent sequences $x$. This space is a linear metric complete space if we introduce the usual definitions of the sum of two elements and of the product of an element by a scalar, and if we define the norm of $x$ by

$$
\|x\|=\sup _{n}\left|x_{n}\right|
$$

Consider the double array of linear functionals on (c) defined by

$$
U_{p q}(x)=\sum_{n=1}^{q} a_{m n} x_{n}, \quad\left(m=m_{p} ; p, q=1,2, \cdots\right) .
$$

Our assumption is that none of the limits

$$
\lim _{q \rightarrow \infty} U_{p q}\left(x^{(p)}\right), \quad(p=1,2, \cdots),
$$

exists. Then, by an important theorem of Banach, * the set $H$ of elements $x$ for which none of the limits

$$
\lim _{q \rightarrow \infty} U_{p q}(x), \quad(p=1,2, \cdots),
$$

exists is of the second category, hence not empty. This means that there exists a convergent sequence $x=\left\{x_{n}\right\}$ such that no series

* S. Banach, Théorie des Opérations Linéaires, Warsaw, 1932, pp. 24-25, Theorem 4. 


$$
\sum_{n=1}^{\infty} a_{m n} x_{n}, \quad \quad\left(m=m_{1}, m_{2}, \cdots\right)
$$

converges. This however cannot occur if $\mathfrak{A}$ is regular in the sense of the definition above, according to which all series

$$
\sum_{n=1}^{\infty} a_{m n} x_{n}, \quad\left(m \geqq m^{\prime}(x)\right),
$$

must converge.

The existence of a fixed $m_{0}$ is thus established, and at the same time it is shown that our modified definition of regularity of $\mathfrak{A}$ is equivalent to the classical one. It is clear that analogous considerations can be applied when instead of summation of series we deal with summation of integrals.

Brown UnIVERSITY

\section{AN EXTENSION TO POLYGAMMA FUNCTIONS OF A THEOREM OF GAUSS*}

BY H. T. DAVIS

1. Introduction. By the polygamma functions we mean the derivatives of $\log \Gamma(x)$, that is, $\Psi(x)=\Gamma^{\prime}(x) / \Gamma(x), \Psi^{\prime}(x), \cdots$, $\Psi^{(n)}(x) . \dagger$ These functions satisfy the difference equations:

$$
\Psi^{(n)}(x+1)-\Psi^{(n)}(x)=(-1)^{n} \frac{n !}{x^{n+1}},
$$

$$
\Psi^{(n)}(1-x)+(-1)^{n+1} \Psi^{(n)}(x)=A_{n}(x), A_{n}(x)=\frac{d^{n}}{d x^{n}}(\pi \operatorname{ctn} \pi x)
$$

subject to the boundary condition,

$$
\Psi^{(n)}(1)=(-1)^{n+1} n ! S_{n+1},
$$

where we employ the abbreviation

$$
S_{m}=1+1 / 2^{m}+1 / 3^{m}+\cdots \cdot
$$

* Presented to the Society, December 27, 1934.

† The name polygamma is suggested by the paper, Tables of the Digamma and Trigamma Functions, by Eleanor Pairman, Tracts for Computers, No. 1, 1919. 